\title{
DISTORTION DISTRIBUTION AND CONVERGENCE ANALYSIS OF SPHERICAL DIAMOND DISCRETE GRIDS
}

\author{
ZHOU Liang-Chen*, LIN Bing-Xian, LV Guo-Nian, ZHAO Zhi-Peng
}

(Key Lab of Virtual Geographic Environment, MOE, Nanjing Normal University, 1\#, Wenyuan Road, Nanjing, Jiangsu Province, P R China, 210023) zhoulch@gmail.com

\begin{abstract}
KEY WORDS: Global Discrete Grid, Spherical Diamond Discrete Grid, Distortion Distribution, Convergence Analysis, Electrical
\end{abstract} Potential Energy

\begin{abstract}
:
Four kinds of subdivision methods of Global Discrete Grid are presented in this paper. Furthermore, we systematically and comprehensively compare and analyze the distortion distribution and convergence of spherical diamond discrete grids. Especially, the Electrical Potential Energy is introduced as index to measure the distortion distribution of spherical diamond discrete grids. The result shows that the properties including the distortion of the area of the grid cell and the distortion of the angle of the grid cell or from the global uniformity of spherical diamond discrete grids which are obtained by recursively bisecting the great arcs on the surface of the globe starting with the icosahedron are much better than the properties of the other three discrete grid obtained by subdivision methods.
\end{abstract}

\section{INTRODUCTION}

Spherical diamond discrete grids are more easily to search neighboring cells and build hierarchical relation, and more suitable to manage global distributed massive spatial data and update local data dynamically, since their geometric structure is similar to the structure of square grids on planar, their coding method is simpler than that of triangular grid or hexagon grid, and they are oriental uniform and radially symmetrical. According to the basic polyhedron (such as, octahedron, icosahedron) and recursive bisection method, foreign and domestic researchers have proposed several different methods to construct spherical rhomboidal discrete grids which are all applied to manage and integrate the global multi-scale and multi-resolution spatial data like great arc bisecting method, longitude and latitude bisecting method, mixed bisecting method based on octahedron, and great arc bisecting method based on icosahedron. The applications based on octahedron include a hierarchical spatial data structure for global geographic information systems (Goodchild, 1922), continuous indexing of hierarchical subdivisions of the globe (BarthildiJ, 2001), spatial data quality and hierarchical comprehensive data model (Dutton, 1999; Gold, 2000;Li, 2003). The applications based on icosahedron are spherical hierarchical data index (Fekete, 1990), global navigation model (Lee, 2000), geodesic discrete global grid system (2003, Sahr).

Getting spherical discrete grid, the geometrical characteristics of which is excellent and distortion distribution of which is uniform, is major subject of the research of Digital Discrete Grid (Goodchild, 2000). But from spherical geometry we know there is a method by which we can get nearly the same instead of absolute the same geometrical characteristics (such as, area, length, and angle) as the planar raster. Before making full use of spherical diamond discrete grids, we need to figure out the questions, such as what's the similarity, what's the regulation of distribution of the area and angle, and are they converge, and analysis the numerical error of the grid model.

\section{GENERATION METHOD OF SPHERICAL DIAMOND DISCRETE GRIDS}

In this paper, we will introduce four kinds of methods by which spherical diamond discrete grids are generated. The grids include spherical diamond discrete grids which are obtained by recursively bisecting the great arcs on the surface of the globe starting with the octahedron, spherical diamond discrete grids which are obtained by recursively bisecting lonlat on the surface of the globe starting with the octahedron, spherical diamond discrete grids which are obtained by mixed method on the surface of the globe starting with the icosahedron and spherical diamond discrete grids which are obtained by recursively bisecting the great arcs on the surface of the globe starting with the icosahedron. They are obtained by projecting regular solids to the surface of sphere. The spherical diamond is combined by two triangles which are generated by bisecting different lines (the great arc, geodesic line or mixed lines) on the surface. Due to the different regular solids and bisecting methods, the geometrical characteristics and distortion convergence of the discrete grids are different. Next we will analyze the characteristics of the spherical diamond discrete grid.

\section{GEOMETRICAL CHARACTERISTICS OF SPHERICAL DIAMOND DISCRETE GRID}

At present, statistical analysis of geometry characteristics of grid cell is the major method used to research the geometry characteristics of the spherical discrete grids. Through analyzing the area of grid cell and major-minor axis ratio of the four kinds of spherical diamond discrete grids by statistical methods, the paper obtains the following conclusions.

(1) The distortion characteristics of the angle and area of the spherical diamond discrete grids which are obtained by recursively bisecting the great arcs on the 
Topics: Global Spatial Grid \& Cloud-based Services

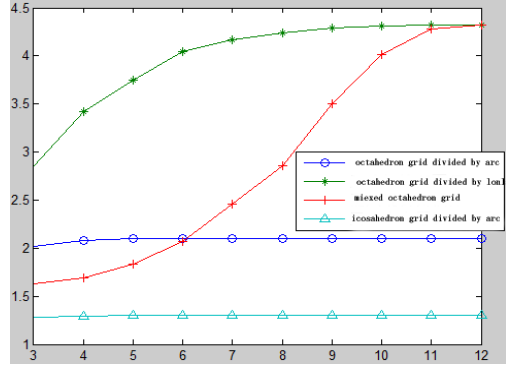

Figure 1. Max/Min area ratio

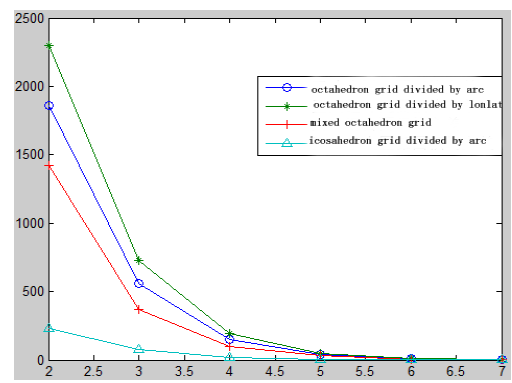

Figure 2. Mean square deviation of $\mathrm{max} / \mathrm{min}$ area ratio

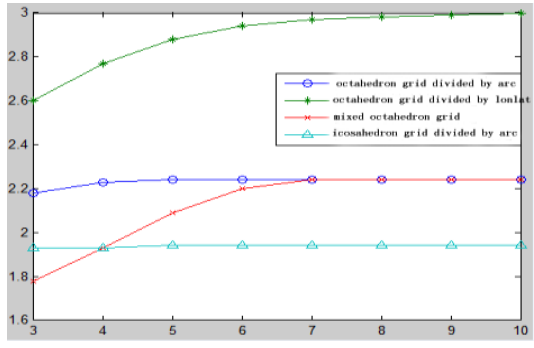

Figure 3. Major-minor axis ratio

must find out what the uniformity of the spherical grids is and how to measure it at first?

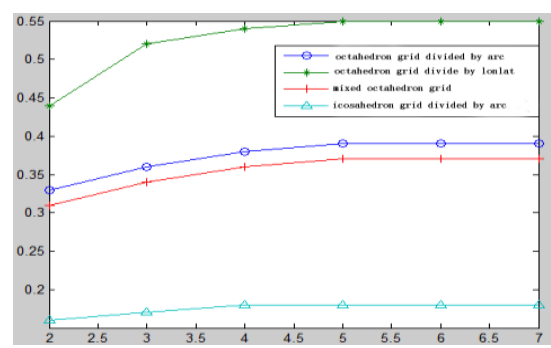

Figure 4. Mean square deviation of major-minor axis ratio

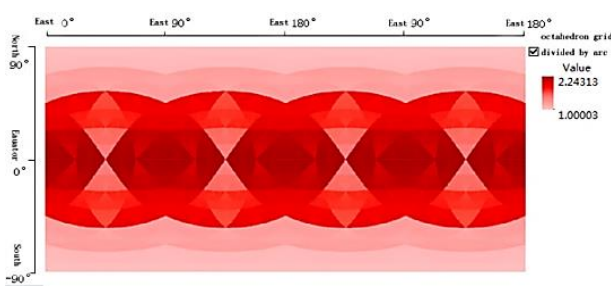

Figure 5 . The planar distribution of octahedron grid divided by $\operatorname{arc}$

surface of the globe starting with the icosahedron are better than others.
(2)

The distortion characteristics of the angle and area of the spherical diamond discrete grids which are

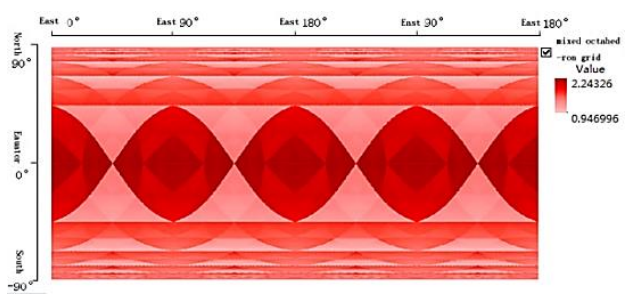

Figure 6 . The planar distribution of octahedron grid divided by mixed method

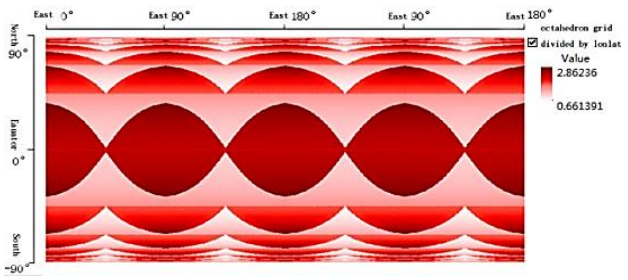

Figure 7. The planar distribution of octahedron grid divided by lonlat

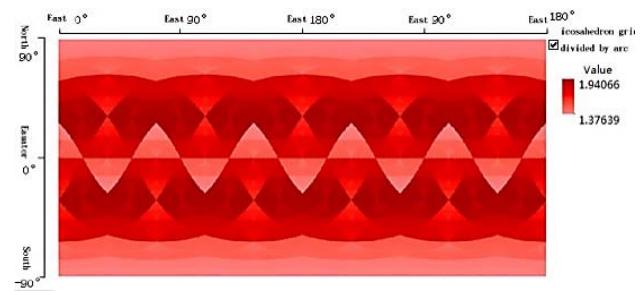

Figure 8 . The planar distribution of icosahedron grid divided by mixed method

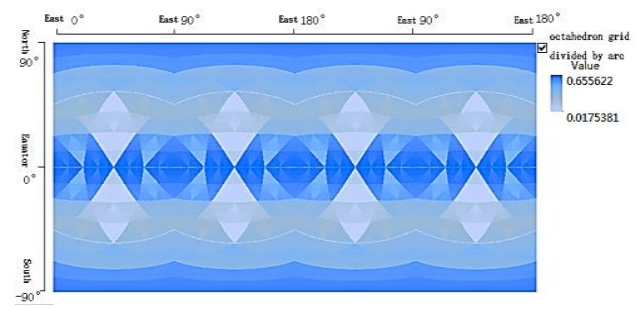

Figure 9. The planar distribution of mean square deviation of major-minor of octahedron grid divided by arc

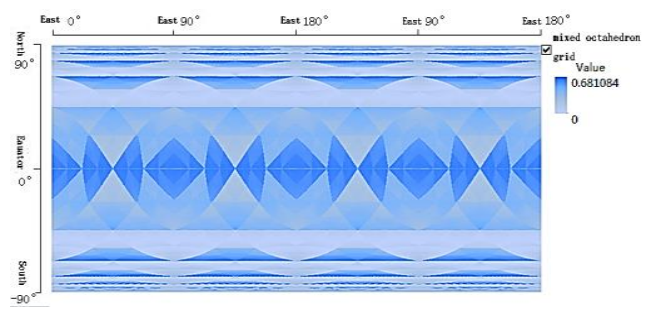

Figure 10. The planar distribution of mean square deviation of major-minor of octahedron grid divided by mixed method

obtained by recursively bisecting the longitude-latitude lines on the surface of the globe starting with the octahedron are worse than others. characteristics of angle of spherical diamond discrete grids 

ISPRS WebMGS 2013 \& DMGIS 2013, 11 - 12 November 2013, Xuzhou, Jiangsu, China

Topics: Global Spatial Grid \& Cloud-based Services

obtained by recursively bisecting arc and spherical diamond discrete grids obtained by recursively mixed bisecting method are the same when $n=7$, but the former is obvious much better than the latter, with regarding to distortion characteristics of area of the grid cell.

Researches above are mainly on the basis of the analysis of geometry characteristics of grid cells. In order to figure out

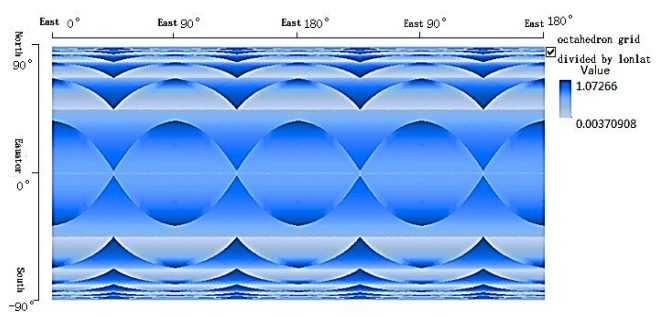

Figure 11. The planar distribution of mean square deviation of major-minor of octahedron grid divided by mixed method

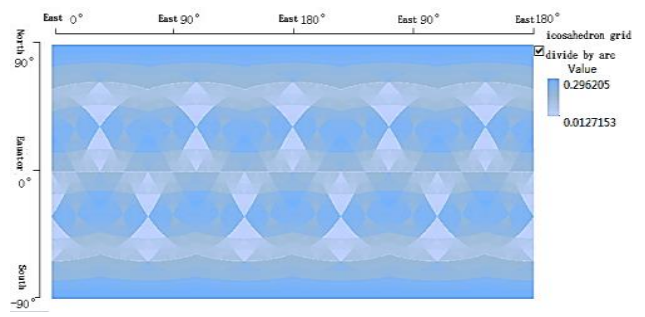

Figure 12. The planar distribution of mean square deviation of major-minor of icosahedron grid divided by arc

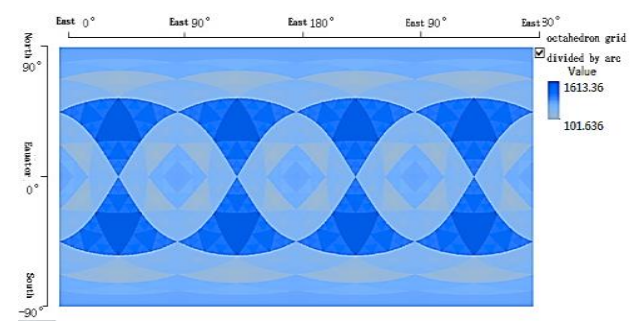

Figure 13. The planar distribution of mean square deviation of major-minor of octahedron grid divided by arc

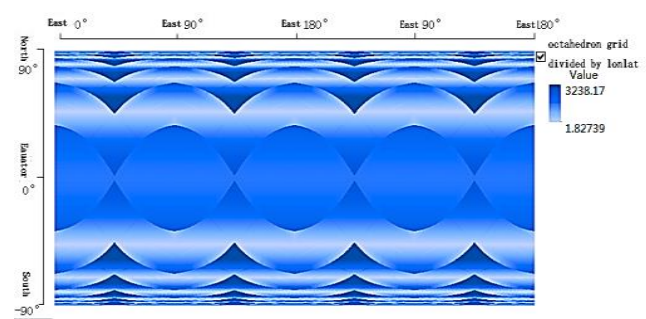

Figure 14. The planar distribution of mean square deviation of major-minor of octahedron grid divided by mixed method

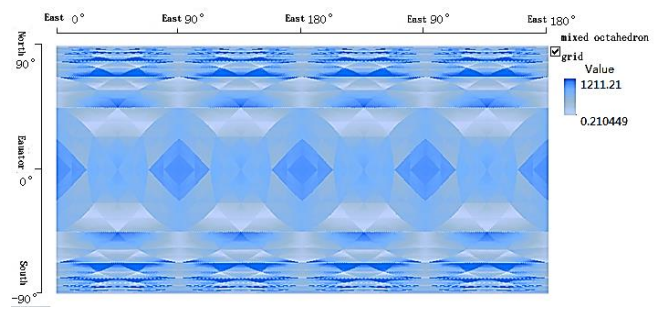

Figure 15. The planar distribution of mean square deviation of major-minor of octahedron grid divided by lonlat

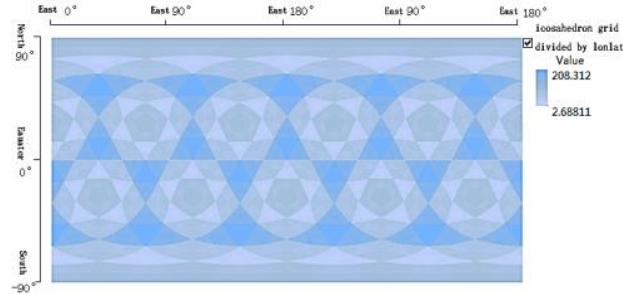

Figure 16. The planar distribution of mean square deviation of major-minor of icosahedron grid divided by arc

the geometry characteristics of the spherical grids comprehensively, we need to analyze the shape of the spherical diamond discrete grids from uniformity in addition to the analysis of the grid cell. Therefore, we

\section{DISTORTION DISTRIBUTION OF SPHERICAI DIAMOND DISCRETE GRID}

\subsection{Electrical Potential Energy}

According to the rule of nature, low energy system is easier to reach steady state. If we put $\mathrm{N}$ electric charges on the rigid spherical surface, the system energy, and the distribution of electric charges if uniform (at least, they are quasi-uniform). The total electrical potential energy equation (Jackson, 1999) is given by

$$
\mathrm{U}(\mathrm{r})=\sum_{\mathrm{i}=1}^{n} \sum_{j=1, j \neq i}^{n} \frac{1}{4 \pi \varepsilon} \frac{q_{1} q_{j}}{r_{4 f}}
$$

Where $r_{i j}$ is the spatial distance between $q_{i}$ and $q_{j}, q_{i}$ is electric quantity of electric charge in the centre of current position, $q_{j}$ is electric quantity of electric charge in grid cells around $q_{i}$.

\subsection{Experimental Analysis}

From the above, we conclude that if we put the electric charges in the centre of the spherical diamond discrete grids, uniform grids have less electrical potential energy. Taking electrical potential energy as evaluation index of uniformity of the grid cells, we can draw following conclusions,

(1) The electrical potential energy and the mean square error of the electrical potential energy of the spherical diamond discrete grids which are obtained by recursively bisecting the great arcs on the surface of the

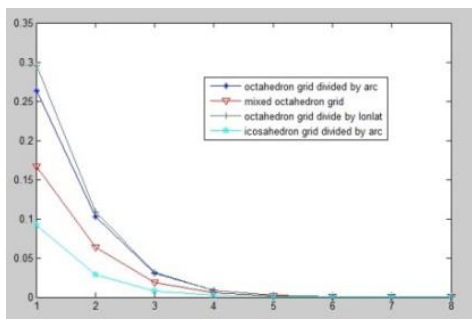

Figure 17. The electrical potential energy of the electrical potential energy 


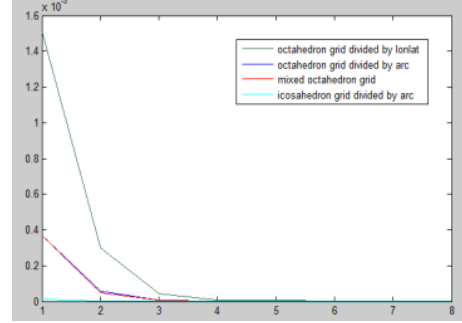

Figure 18. Mean square error of the electrical potential energy of the spherical diamond discrete grids

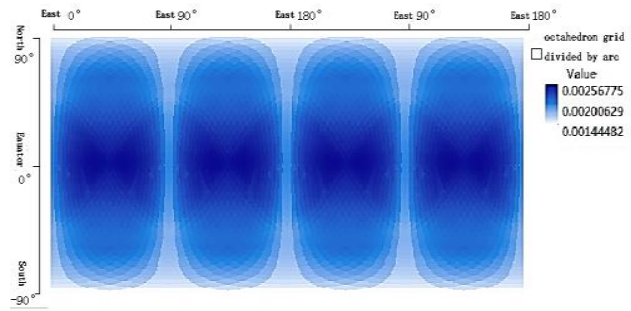

Figure 19. Distribution of electrical potential energy of spherical diamond discrete grids octahedron grid divided by arc

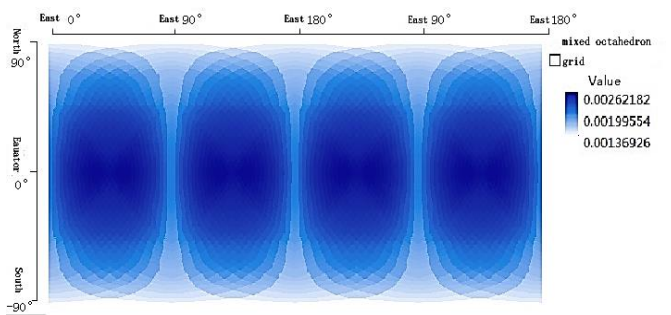

Figure 20. Distribution of electrical potential energy of spherical diamond discrete grids octahedron grid divided by mixed method

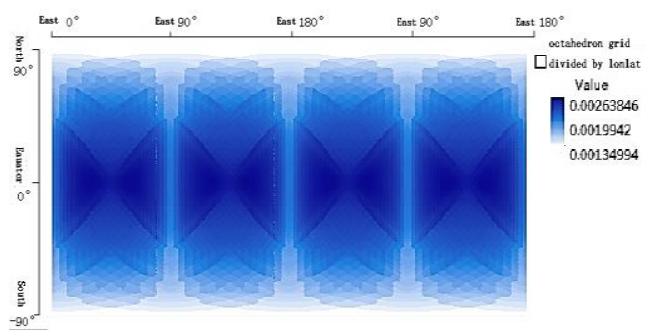

Figure 21. Distribution of electrical potential energy of spherical diamond discrete grids octahedron grid divided by lonlat

are obtained by recursively bisecting the great arcs on the surface of the globe starting with the icosahedron is the best.

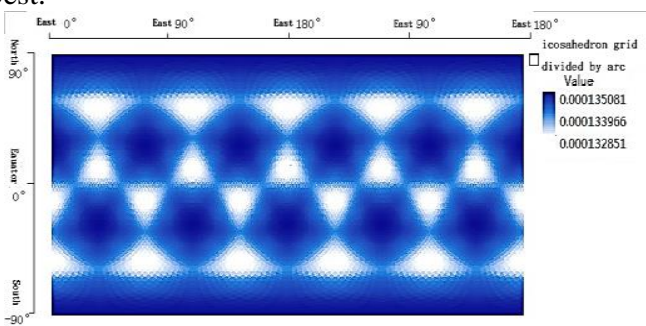

Figure 22. Distribution of electrical potential energy of spherical diamond discrete grids icosahedron grid divided by arc globe starting with the icosahedron are lowest, they demonstrates that the uniformity of the spherical diamond discrete grids which

(2) The longitude-latitude lines on the surface of the globe starting with the octahedron are the highest, they demonstrate that the uniformity of spherical diamond

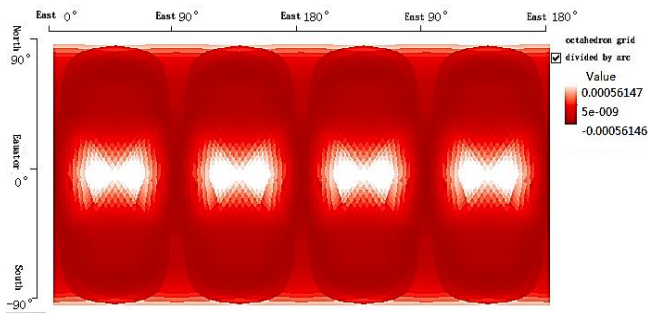

Figure 21. Distribution of electrical potential energy of spherical diamond discrete grids octahedron grid divided by arc

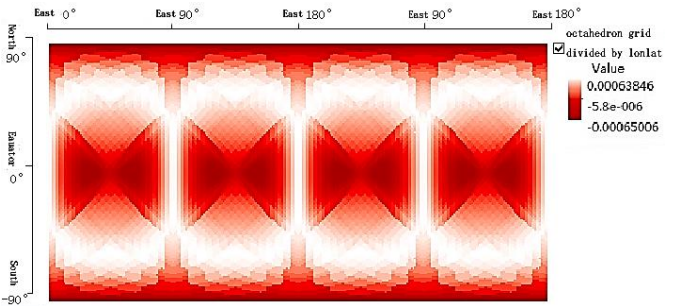

Figure 22. Distribution of electrical potential energy of spherical diamond discrete grids octahedron grid divided by lonlat

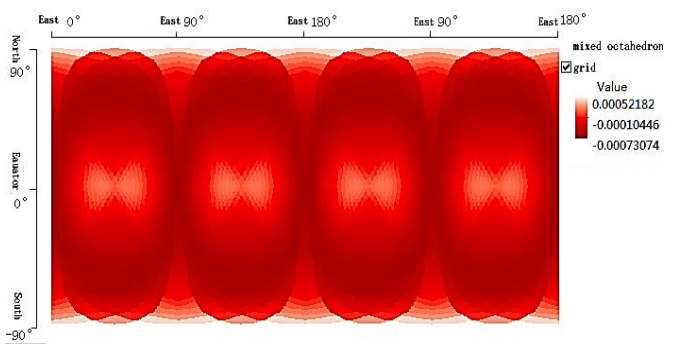

Figure 23. Distribution of electrical potential energy of spherical diamond discrete grids octahedron grid divided by mixed method

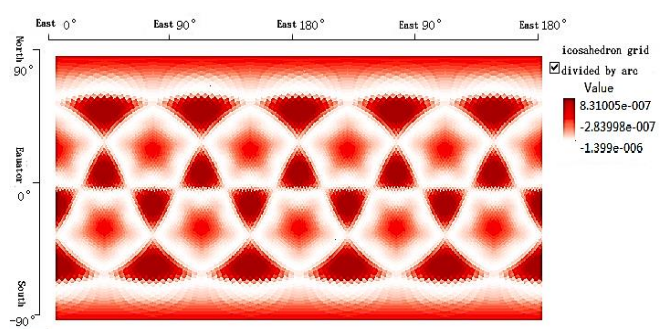

Figure 24. Distribution of electrical potential energy of spherical diamond discrete grids icosahedron grid divided by $\operatorname{arc}$

discrete grids which are obtained by recursively bisecting the longitude-latitude lines on the surface of the globe starting with the octahedron are the highest, they demonstrates that the uniformity of spherical diamond discrete grids which are obtained by recursively bisecting the longitude-latitude lines on the surface of the globe starting with the octahedron is worst. 
The electrical potential energy of spherical diamond discrete grids obtained by recursively bisecting arc and spherical diamond discrete grids obtained by recursively bisecting the longitude-latitude lines on the surface of the globe starting with the octahedron are nearly the same, but the mean square error of the electrical potential energy of former is obvious much better than that of the latter. The mean square error of the electrical potential energy of spherical diamond discrete grids obtained by recursively bisecting arc and spherical diamond discrete grids obtained by recursively mixed bisecting method on the surface of the globe starting with the octahedron are nearly the same, but the electrical potential energy of the former is lower than the latter, it demonstrates that the latter is steady during bisecting recursively.

\section{CONCLUSIONS}

Bases on the above analysis, we can see that the spherical diamond discrete grids which are obtained by recursively bisecting the great arcs on the surface of the globe starting with the icosahedron are better than the others whether in distortion of the area of the grid cell and the distortion of the angle of the grid cell or from the global uniformity. Furthermore, the paper presents a measure method based on electrical potential energy of analyzing the global uniformity of the grids; the method can also be used to research the geometry characteristics of all the other kinds' grids.

\section{ACKNOWLEDGEMENT}

This research is sponsored by National Natural Science Foundation of China (41001224) and A Project Funded by the Priority Academic Program Development of Jiangsu Higher Education Institutions

\section{REFERENCES}

BarthildiJ,III.2001.Continuous indexing of hierarchica lsubdivisionsofthe globe.Int.J.Geographical Information Science.15(6).pp489-522.

Dutton,G.1999.Scale, sinuosity, and point selection in digital line generalization.Cartography and Geographic Informatiom Science.26(1).pp33-53.

Dutton,G.1996.Encoding and handling geospatial data with hierarchical triangular meshes.Proceeding of 7th International Symposium on Spatial Data Handling.In:Kraak MJ,Molenaar $M(E d.) . p p 34-43$.

Fekete.1990. Rendering and managing spherical data with sphere quadtrees. Proceedings of the 1st conference on Visualization '90. IEEE Computer Society Press.pp176-186.

Goodchild, Michael F. and Shiren, Yang. 1992.A hierarchical spatial data structure for global geographic information systems. CVGIP: Graph. Models Image Process.54(1),pp31-44.

Goodchild, Michael F.2000.Discrete global grids for digital earth.International Conference on Discrete Global Grids.California:Santa Barbara.
Gold,CM.2000.Towards the Global GIS.ISPRS Journal of Photogrammetry and Remote Sensing.55(3).pp150-163.

Jackson,JohnDavid.1999.Classical Electrodynamic. 3rd.USA: John Wiley \& Sons.pp. 40-43.

Lee M, Samet H.2000.Navigating through triangle meshes implemented as linear quadtree.ACM transactions on Graphics.19(2).pp79-121.

Sahr,K.2003.Geodesic discrete global grid system.Cartography and Geographic Information Science.30(2).pp121-134.

Li,DR.2003.From digital map to spatial information grid.Journal of Wuhan University(Edition of Information Science).28(6).pp642-650. 